Review

\title{
Chlamydia pneumoniae and Oxidative Stress in Cardiovascular Disease: State of the Art and Prevention Strategies
}

\author{
Marisa Di Pietro, Simone Filardo, Fiorenzo De Santis, Paola Mastromarino and Rosa Sessa * \\ Department of Public Health and Infectious Diseases, "Sapienza" University, Rome 00185, Italy; \\ E-Mails: marisa.dipietro@uniroma1.it (M.D.P.); simone.filardo@uniroma1.it (S.F.); \\ fiorenzo.desantis@uniroma1.it (F.D.S.); paola.mastromarino@uniroma1.it (P.M.) \\ * Author to whom correspondence should be addressed; E-Mail: rosa.sessa@uniroma1.it; \\ Tel.: +39-64-991-4102; Fax: +39-64-991-4634.
}

Academic Editor: Francis Miller Jr.

Received: 4 November 2014 / Accepted: 26 December 2014 / Published: 30 December 2014

\begin{abstract}
Chlamydia pneumoniae, a pathogenic bacteria responsible for respiratory tract infections, is known as the most implicated infectious agent in atherosclerotic cardiovascular diseases (CVDs). Accumulating evidence suggests that C. pneumoniae-induced oxidative stress may play a critical role in the pathogenesis of CVDs. Indeed, the overproduction of reactive oxygen species (ROS) within macrophages, endothelial cells, platelets and vascular smooth muscle cells (VSMCs) after C. pneumoniae exposure, has been shown to cause low density lipoprotein oxidation, foam cell formation, endothelial dysfunction, platelet adhesion and aggregation, and VSMC proliferation and migration, all responsible for the typical pathological changes of atherosclerotic plaque. The aim of this review is to improve our insight into $C$. pneumoniae-induced oxidative stress in order to suggest potential strategies for CVD prevention. Several antioxidants, acting on multi-enzymatic targets related to ROS production induced by $C$. pneumoniae, have been discussed. A future strategy for the prevention of $C$. pneumoniae-associated CVDs will be to target chlamydial HSP60, involved in oxidative stress.
\end{abstract}

Keywords: C. pneumoniae; cardiovascular disease; oxidative stress; prevention strategies 


\section{Introduction}

Cardiovascular disease (CVD) is a major health problem in developed countries with over 17 million deaths per year [1] and the main pathological process underlying this disease is the atherosclerosis.

Atherosclerosis typically begins with endothelial injury followed by low-density lipoprotein (LDL) oxidation and accumulation within vascular cells, triggering the pro-inflammatory cascade [interleukin (IL)-1, IL-6 and tumor necrosis factor (TNF)- $\alpha$ ] and the subsequent proliferation of smooth muscle cells. Through this complex process, a sequence of events, including foam cell formation followed by fibrous cap and thrombus formation in the advanced plaque occurs leading to cardiovascular diseases, such as coronary heart diseases (angina, myocardial infarction), stroke, and peripheral vascular diseases [2].

Current opinion is that the most implicated infectious agent in the pathogenesis of CVDs is Chlamydia pneumoniae, known as the etiologic agent of respiratory tract infections $[3,4]$. C. pneumoniae is an intracellular obligate pathogen with a unique developmental cycle, characterized by two alternating functionally and morphologically distinct forms: The elementary body, the metabolically inert and infectious form, and the reticulate body, the intracellular replicative form. In the last years, the attention has been drawn to a third non-replicating and non-infectious form, called persistent form, described as involved in the pathogenesis of chronic inflammatory diseases such as atherosclerosis. Indeed, chlamydial persistent form may endure for a long time inside host cells, since it is able to evade the host immune response leading to a chronic inflammatory state in the vascular wall [5-7].

The relationship between C. pneumoniae and CVDs has been first suggested in 1988 by Saikku et al. [8]. Since then, increasing evidence has supported the involvement of $C$. pneumoniae in the pathogenesis of CVDs. The association has been well documented by seroepidemiological studies $[4,9,10]$, direct detection of microorganism within atherosclerotic plaque [9,11-13] and in vivo studies showing the ability of $C$. pneumoniae to disseminate from lungs to extrapulmonary sites, such as the vascular wall, by peripheral blood mononuclear cells [14-17]. Stronger evidence on the matter came, mainly, from the isolation of viable C. pneumoniae from the atheroma [18-20] and by experimental studies showing an atherosclerotic lesion exacerbation following the $C$. pneumoniae inoculation of hyperlipidemic animal models [21-24].

Despite extensive evidence on the association between $C$. pneumoniae and CVDs, there are still issues to be addressed, such as the lack of well-standardized and adequately validated diagnostic tests for detecting C. pneumoniae. This problem is made more difficult for the presence of $C$. pneumoniae persistent forms, which are not consistently detectable by currently used methods. Surely, in the future, a deep and complete analysis of the genome of $C$. pneumoniae from CVD patients will more likely increase our understanding of the pathogenetic mechanisms underlying the development of atherosclerosis.

In fact, the mechanisms by which $C$. pneumoniae may influence the atherogenesis have yet to be fully clarified. Initially, it has been hypothesized that $C$. pneumoniae may contribute to atherosclerosis through inflammation, as evidenced by an increased production of inflammatory cytokines (IL1- $\beta$, IL-6, IL-8 and TNF- $\alpha$ ) and chemokines found in vascular cells involved in the atherosclerotic process [25-30]. This hypothesis has been further confirmed by increased levels of inflammatory markers, observed in patients with CVDs and C. pneumoniae infection [31-33]. 
More recently, it is believed that $C$. pneumoniae may contribute to atherosclerosis through oxidative stress, as suggested by an increased production of reactive oxygen species (ROS) following C. pneumoniae interaction with vascular cells [34].

The oxidative stress, characterized by ROS overproduction, was demonstrated for the first time in C. pneumoniae infected macrophages, leading to LDL oxidation and foam cell formation. The increased production of ROS, induced by C. pneumoniae, has also been demonstrated in platelets, endothelial cells and vascular smooth muscle cells (VSMCs) causing both LDL oxidation and further pro-atherogenic effects, such as endothelial dysfunction, VSMC proliferation and migration, and platelet adhesion and aggregation [34].

In addition, there is also the evidence that $C$. pneumoniae and oxidized LDL (oxLDL) induce necrosis in macrophages as well as in endothelial cells, thus contributing to vascular inflammation and progression of atherosclerotic lesion $[35,36]$.

The growing body of evidence linking $C$. pneumoniae to atherosclerosis through the elicitation of oxidative stress in the vascular wall raises interesting questions concerning the possibilities of CVD prevention by limiting ROS production and/or accelerating their inactivation. Therefore the aim of this review is to improve our insight into C. pneumoniae-induced oxidative stress in order to suggest potential strategies for the prevention of CVDs.

\section{Chlamydia pneumoniae-Induced Oxidative Stress}

In the vascular wall, ROS are produced by several enzyme systems including NADPH oxidase (NOX), xanthine oxidase (XO), uncoupled endothelial nitric oxide synthase (eNOS) and the mitochondrial electron transport chain. On the other hand, the vasculature is protected by antioxidant enzyme systems, including superoxide dismutases (SOD), catalase, glutathione peroxidases (GPx) and paraoxonases, which detoxify ROS. ROS, including free oxygen radicals, oxygen ions and peroxides, under physiological conditions, act as signaling molecules and play an important role in the regulation of vascular tone, cell growth and proliferation, apoptosis, and inflammatory responses. When the release of ROS is not limited by antioxidant defense systems, oxidative stress promotes atherogenesis through several mechanisms, including lipoprotein and phospholipid oxidation [37,38].

Generally speaking, macrophages, endothelial cells, VSMCs and platelets involved in atherosclerotic process may all be a source of ROS through different enzymatic pathways [38]. In macrophages, endothelial cells and VSMCs, ROS are produced by NOX activity and mitochondrial respiratory electron transport chain. In endothelial cells, under pathological conditions associated to oxidative stress, ROS are also produced by uncoupled eNOS activity, potentiating the preexisting oxidative stress. Lastly, in platelets, ROS production seems to depend on cyclooxygenase (COX), lipoxygenase (LOX), NOX and nitric oxide synthase (NOS) activities [39].

An enhanced ROS accumulation in all the cells involved in the atherosclerotic process has been demonstrated following the exposure to C. pneumoniae [34] (Figure 1). 


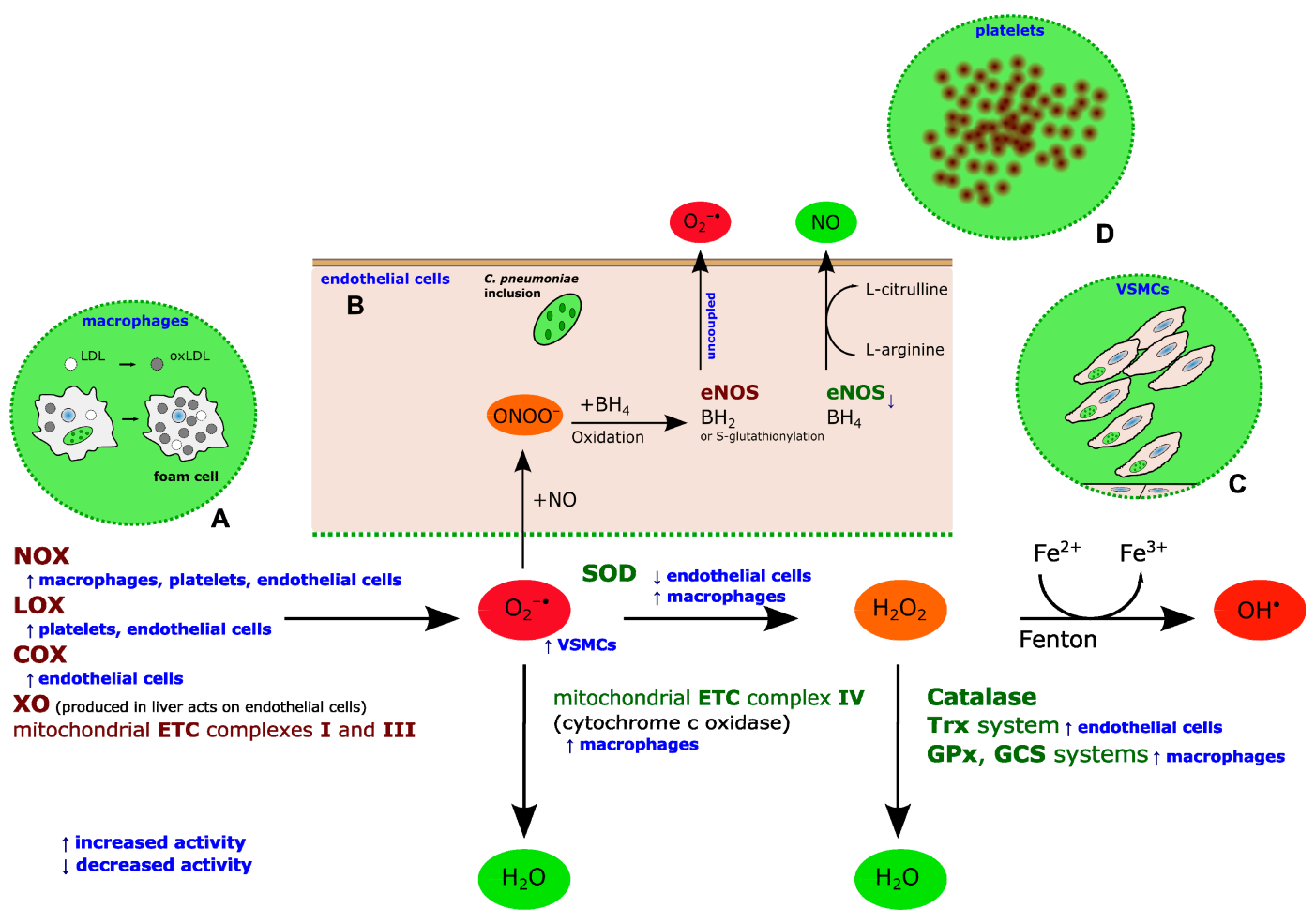

Figure 1. ROS generation and inactivation in the vascular wall and the contribution of $C$. pneumoniae. Generally, superoxide anion $\left(\mathrm{O}_{2}^{-\cdot}\right)$ can be produced by NADPH oxidase (NOX), lipoxygenases (LOX), cycloxygenases (COX), xanthine oxidase (XO) and mitochondrial electron transport chain (ETC) complexes I and III during the oxidative respiration. $\mathrm{O}_{2}^{-\cdot}$ can be disproportionated to hydrogen peroxide $\left(\mathrm{H}_{2} \mathrm{O}_{2}\right)$ by superoxide dismutase (SOD). $\mathrm{H}_{2} \mathrm{O}_{2}$ can spontaneously convert to hydroxyl radical $\left(\mathrm{OH}^{\prime}\right)$ via the Fenton reaction. Alternatively, $\mathrm{H}_{2} \mathrm{O}_{2}$ can be detoxified to water via catalase, glutathione peroxidase (GPx) and $\gamma$ glutamylcysteine synthase (GCS) or thioredoxin (Trx) systems. $\mathrm{O}_{2}{ }^{-\cdot}$ can also be directly detoxificated into water by cytochrome c oxidase (mitochondrial ETC complex IV). Under pathological conditions associated to oxidative stress, superoxide anion $\left(\mathrm{O}_{2}^{--}\right)$may react with nitric oxide (NO), produced by endothelial nitric oxide synthase (eNOS), yielding peroxynitrite $\left(\mathrm{ONOO}^{-}\right)$, which oxidizes the tetrahydrobiopterin $\left(\mathrm{BH}_{4}\right)$ cofactor. Therefore, the $\mathrm{BH}_{4}$ deficiency leads to eNOS uncoupling with consequent release of $\mathrm{O}_{2}^{-\cdot}$ instead of NO. In addition, under oxidative stress eNOS uncoupling may occur following to eNOS $\mathrm{S}$ glutathionylation. C. pneumoniae promotes oxidative stress through different enzymatic pathways: in macrophages, it enhances both NOX activity and activities of detoxification systems such as mitochondrial ETC complex IV (cytochrome c oxidase), SOD, GPx and GCS; in platelets, it enhances NOX and LOX activities; in VSMCs, ROS production is elicited in a NOX-independent way; in endothelial cells, it enhances NOX, LOX and COX activities, while reducing SOD, Trx and eNOS activities. Blue arrows refer to C. pneumoniae effect on the related enzyme activity. Overall, C. pneumoniae-induced oxidative stress contributes to atherogenesis, leading to: (A) intracellular survival of $C$. pneumoniae inside macrophages, LDL oxidation and foam cell formation; (B) endothelial dysfunction, characterized by increased production of anion superoxide and reduced NO bioavailability; (C) VSMC proliferation and migration; and (D) platelet activation and aggregation. 
In macrophages, $C$. pneumoniae has been shown to elicit superoxide anion production via NOX pathway and, at the same time, increase the antioxidant activity of cytochrome c oxidase, which may paradoxically attenuate the ROS release. In particular, it has been demonstrated that $C$. pneumoniae stimulated NOX and cytochrome c oxidase, whose expression is dependent on the binding of this microorganism to macrophage CD14 receptors and $\mathrm{Ca}^{2+}$ influx signaling [40]. Despite ROS production, $C$. pneumoniae, unlike the majority of pathogens, has been demonstrated to survive in macrophages by maintaining a relatively high antioxidant:oxidant ratio, thereby abrogating the bacteria-killing effect of ROS. The ability of $C$. pneumoniae to limit ROS production in macrophages is also supported by the evidence that this microorganism up-regulates the activities of several antioxidant enzyme systems, such as SOD, GPx and $\gamma$-glutamylcysteine synthase ( $\gamma$-GCS) [41].

In endothelial cells, the increased production of superoxide anion may be a consequence of increased expression of COX-2, NOX-2 and NOX-4 paralleled by down-regulation of SOD-1 and thioredoxin-1 following the exposure to $C$. pneumoniae [42]. A similar mechanism has also been observed after the exposure to chlamydial heat shock protein-60 (cHSP60), a known virulence factor of $C$. pneumoniae. cHSP60 has been shown to contribute to endothelial dysfunction by decreasing eNOS expression and NO production through several regulatory mechanisms, including oxidative stress $[43,44]$. Reduced NO synthesis has also been implicated in cHSP60-induced dysfunction of endothelial progenitor cells, contributing to atherosclerotic lesion progression by impairing vascular repair processes [45]. Lastly, cHSP60 may play a critical role in ox-LDL uptake of endothelial cells, which results from elevated expression of lectin-like oxidized low density lipoprotein receptor 1 (LOX-1) mediated by different molecular pathways, such as NOX activation and eNOS activation phosphatidylinositol-4,5-bisphosphate 3-kinase (PI3K)-protein kinase B (Akt) dependent [46].

In VSMCs, C. pneumoniae elicits ROS production in a manner that is independent from NOX activity. Moreover, ROS, largely secreted in the extracellular compartment, affect other vascular cells, inactivating the vasoprotective molecule NO and, thus, contributing to the onset of endothelial dysfunction [47].

At last, in platelets, $C$. pneumoniae stimulates ROS generation and secretion through COX activation and LOX and NOS activation, mediated by protein kinase C (PKC). It has been hypothesized that $C$. pneumoniae, via the lipopolysaccharide, may interact with platelet surface structures and, thus, trigger PKC activity, which, in turn, phosphorylates proteins involved in shape change, aggregation, and ROS production [39].

\section{C. Pneumoniae-Induced Oxidative Stress as a Target for CVD Prevention}

At the end of 1990s, the wide number of studies, showing the involvement of C. pneumoniae in the pathogenesis of atherosclerosis and CVDs, has opened up new opportunities in the management of adverse cardiovascular outcomes. As a result, numerous clinical antibiotic trials for the prevention of cardiovascular events have been undertaken, but most of them have failed to demonstrate any benefit of anti-chlamydial treatment $[3,48]$. The failure of clinical trials has been attributed to several factors, as the refractoriness of chlamydial chronic infection to antibiotics, the enrolment of subjects with advanced cardiovascular disease, etc. $[3,49]$. Actually, the lack of beneficial effects on cardiovascular events in clinical antibiotic trials may also be explained by current knowledge on $C$. pneumoniae induced oxidative stress in vascular wall. 
Therefore, an approach targeting $C$. pneumoniae-induced oxidative stress by preventing ROS generation may be an attractive strategy for CVD prevention.

To date, several antioxidant compounds have been proposed to prevent the ROS-mediated pro-atherogenic effects, including, as previously reported, LDL oxidation, endothelial dysfunction, VSMC proliferation and migration, and platelet activation induced by $C$. pneumoniae infection.

For example, vitamin $\mathrm{E}$ has been shown to prevent the inflammatory state by inhibiting ROS production in endothelial cells, following the exposure to C. pneumoniae and oxLDL [36].

Based on the same principle, compounds, such as curcumin and resveratrol, have been considered as possible inhibitors of the enzyme systems generating ROS in the vascular wall. In C. pneumoniae-infected monocytes, curcumin and resveratrol have been described as preventing the NOX-mediated ROS production by inhibiting protein kinase $\mathrm{C}$; Resveratrol has also been shown to inhibit ROS production by directly decreasing NOX activity [50]. The effectiveness of resveratrol in preventing the atherogenic changes induced by $C$. pneumoniae has been confirmed in our previous study, as evidenced by the relevant decrease in superoxide anion and oxLDL production, and in the number of foam cells [51].

Also, COX-2 inhibitors such as ibuprofen and diclofenac have been found to reduce C. pneumoniae-induced ROS production in monocytes so that they might limit the LDL oxidation as well as the foam cell formation [52].

Interestingly, other investigators have focused their attention on lipid lowering drugs such as statins (coenzyme A reductase inhibitors), since they do not only reduce LDL levels but show antioxidant activity as well [53]. Cerivastatin has been demonstrated to inhibit $C$. pneumoniae-induced oxidative stress in macrophages, VSMCs as well as endothelial cells, thus preventing the inflammation and consequently the atherosclerosis development and progression [54,55]. Fluvastatin has been shown to limit oxLDL uptake in C. pneumoniae-infected endothelial cells by inhibiting LOX-1 scavenger receptor activity, which is partially regulated by ROS [56], and, hence, preventing the endothelial dysfunction and progression of atherosclerotic lesion.

An alternative strategy for the prevention of oxidative stress may be to enhance the anti-oxidant cellular defenses [57]. Among the substances able to mimic the biochemical activity of SOD, Mn (III) tetrakis (4-benzoic acid) porphyrin chloride (MnTBAP) has been found to stimulate NOS activity in endothelial cells, down-regulated after the exposure to cHSP60, thus improving the endothelial function by reducing ROS levels and increasing NO bioavailability [43]. Similarly, other free radical scavengers, such as sesamol, have been shown to be effective in inhibiting the proliferation of VSMCs induced by cHSP60 [58].

Nevertheless, concerning the general activity of antioxidants in CVD prevention, early studies have reported encouraging effects, whereas large randomized clinical trials have showed detrimental effects. More recently, a meta-analysis has showed that there is no evidence to support the use of vitamins for the prevention of CVDs [59].

Also, it should be noticed that some antioxidants, mainly vitamins such as vitamin E, may have potential pro-oxidant effects above a certain concentration due to the accumulation of $\alpha$-tocopherol radicals [60].

Overall, further intense investigations are needed to garner a more complete picture of the effectiveness of these compounds in the prevention of CVDs associated to C. pneumoniae. Actually, the existence of numerous antioxidants acting on multi-enzymatic targets involved in oxidative stress makes 
the identification of effective molecules for the prevention of $C$. pneumoniae-associated CVDs more difficult and demanding.

Therefore an anti-virulence strategy, already considered for the management of infections, may be an intriguing perspective for the prevention of CVDs related to C. pneumoniae-mediated oxidative stress. The anti-virulence strategy consists in identifying inhibitors able to specifically target the virulence determinants involved in pathogenetic processes. Strategies that target virulence factors have already been developed for other pathogens, such as, for example, compounds blocking the type III secretion system of Chlamydia trachomatis, Escherichia coli, etc., or interfering with the quorum sensing of Pseudomanas aeruginosa, Staphylococcus aureus, etc. [61-63]. This approach may pave the way to new molecules targeting the virulence determinants involved in $C$. pneumoniae-induced oxidative stress, thus inhibiting the development and progression of atherosclerotic process.

Concerning chlamydial virulence factors, C. pneumoniae HSP60, a protein produced by reticulate bodies in chronic infection, has been demonstrated to contribute to LDL oxidation and endothelial dysfunction through oxidative stress $[42,43,64]$ as well as to stimulate VSMC proliferation and inflammatory responses through Toll like receptor activation [65-67]. cHSP60 may represent, then, a potential target for prevention of CVDs associated to $C$. pneumoniae-induced oxidative stress.

\section{Conclusions}

C. pneumoniae-induced oxidative stress seems to play a crucial role in the pathogenesis of CVDs. The ROS overproduction observed in macrophages, endothelial cells, VSMCs and platelets following the exposure to $C$. pneumoniae is thought to contribute to the initiation, progression and rupture of lipid-rich vascular lesion. A future strategy will be to target chlamydial HSP60, involved in oxidative stress, in order to identify promising candidates for the prevention of $C$. pneumoniae-associated CVDs.

\section{Acknowledgments}

This study was supported by grants from Center for Social Disease Research, "Sapienza” University Rome, to Rosa Sessa.

\section{Author Contributions}

All authors contributed to conception and design, and gave final approval of the version to be published. Marisa Di Pietro, Simone Filardo, Fiorenzo De Santis, Paola Mastromarino, Rosa Sessa participated to its design and coordination and helped to draft the manuscript; Fiorenzo De Santis projected and designed the schematic picture; Marisa Di Pietro and Rosa Sessa have been involved in critical revision of the manuscript.

\section{Conflicts of Interest}

The authors declare no conflict of interest. 


\section{References}

1. Mendis, S.; Puska, P.; Norrving, B.; World Health Organization; World Heart Federation; World Stroke Organization. Global Atlas on Cardiovascular Disease Prevention and Control; Mendis S., Puska P., Norrving B., Eds.; Available online: http://whqlibdoc.who.int/publications/ 2011/9789241564373_eng.pdf (accessed on 2011).

2. Laboz, C.; Mostaza, J.M. Atherosclerosis as a systemic disease. Rev. Esp. Cardiol. 2007, 60, 184-195.

3. Rosenfeld, M.E.; Campbell, L.A. Pathogens and atherosclerosis: Update on the potential contribution of multiple infectious organisms to the pathogenesis of atherosclerosis. Thromb. Haemost. 2011, 106, 858-867.

4. Sessa, R.; di Pietro, M.; Filardo, S.; Turriziani, O. Infectious burden and atherosclerosis: A clinical issue. World J. Clin. Cases 2014, 2, 240-249.

5. Hogan, R.J.; Mathews, S.A.; Mukhopadhyay, S.; Summersgill, J.T.; Timms, P. Chlamydial persistence: Beyond the biphasic paradigm. Infect. Immun. 2004, 72, 1843-1855.

6. Schoborg, R.V. Chlamydia persistence-A tool to dissect chlamydia-Host interactions. Microbes Infect. 2011, 13, 649-662.

7. Di Pietro, M.; Tramonti, A.; de Santis, F.; de Biase, D.; Schiavoni, G.; Filardo, S.; Zagaglia, C.; Sessa, R. Analysis of gene expression in penicillin G induced persistence of Chlamydia pneumoniae. J. Biol. Regul. Homeost. Agents 2012, 26, 277-284.

8. Saikku, P.; Leinonen, M.; Mattila, K.; Ekman, M.R.; Nieminen, M.S.; Mäkelä, P.H.; Huttunen, J.K.; Valtonen, V. Serological evidence of an association of a novel Chlamydia, TWAR, with chronic coronary heart disease and acute myocardial infarction. Lancet 1988, 2, 983-986.

9. Joshi, R.; Khandelwal, B.; Joshi, D.; Gupta, O.P. Chlamydophila pneumoniae infection and cardiovascular disease. N. Am. J. Med. Sci. 2013, 5, 169-181.

10. Sakurai-Komada, N.; Iso, H.; Koike, K.A.; Ikeda, A.; Umesawa, M.; Ikehara, S.; Inoue, M.; Tsugane, S. Association between Chlamydophila pneumoniae infection and risk of coronary heart disease for Japanese: The JPHC study. Atherosclerosis 2014, 233, 338-342.

11. Sessa, R.; di Pietro, M.; Schiavoni, G.; Petrucca, A.; Cipriani, P.; Zagaglia, C.; Nicoletti, M.; Santino, I.; del Piano, M. Measurement of Chlamydia pneumoniae bacterial load in peripheral blood mononuclear cells may be helpful to assess the state of chlamydial infection in patients with carotid atherosclerotic disease. Atherosclerosis 2007, 195, e224-e230.

12. Atik, B.; Johnston, S.C.; Dean, D. Association of carotid plaque Lp-PLA(2) with macrophages and Chlamydia pneumoniae infection among patients at risk for stroke. PLoS One 2010, 5, e11026.

13. Luque, A.; Turu, M.M.; Rovira, N.; Juan-Babot, J.O.; Slevin, M.; Krupinski, J. Early atherosclerotic plaques show evidence of infection by Chlamydia pneumoniae. Front. Biosci. 2012, 4, 2423-2432.

14. Moazed, T.C.; Kuo, C.C.; Grayston, J.T.; Campbell, L.A. Evidence of systemic dissemination of Chlamydia pneumoniae via macrophages in the mouse. J. Infect. Dis. 1998, 177, 1322-1325.

15. Sessa, R.; di Pietro, M.; Schiavoni, G.; Santino, I.; Benedetti-Valentini, F.; Perna, R.; Romano, S.; del Piano, M. Chlamydia pneumoniae DNA in patients with symptomatic carotid atherosclerotic disease. J. Vasc. Surg. 2003, 37, 1027-1031. 
16. Di Pietro, M.; Filardo, S.; Cazzavillan, S.; Segala, C.; Bevilacqua, P.; Bonoldi, E.; D’Amore, E.S., Rassu M.; Sessa R. Could past Chlamydial vascular infection promote the dissemination of Chlamydia pneumoniae to the brain? J. Biol. Regul. Homeost. Agents 2013, 27, 155-164.

17. Di Pietro, M.; Schiavoni, G.; Sessa, V.; Pallotta, F.; Costanzo, G.; Sessa, R. Chlamydia pneumoniae and osteoporosis-associated bone loss: A new risk factor? Osteoporos. Int. 2013, 24, 1677-1682.

18. Ramirez, J.A. Isolation of Chlamydia pneumoniae from the coronary artery of a patient with coronary atherosclerosis. The Chlamydia pneumoniae/Atherosclerosis Study Group. Ann. Intern. Med. 1996, 125, 979-982.

19. Maass, M.; Bartels, C.; Engel, P.M.; Mamat, U.; Sievers, H.H. Endovascular presence of viable Chlamydia pneumoniae is a common phenomenon in coronary artery disease. J. Am. Coll. Cardiol. 1998, 31, 827-832.

20. Apfalter, P.; Loidl, M.; Nadrchal, R.; Makristathis, A.; Rotter, M.; Bergmann, M.; Polterauer, P.; Hirschl, A.M. Isolation and continuous growth of Chlamydia pneumoniae from arterectomy specimens. Eur. J. Clin. Microbiol. Infect. Dis. 2000, 19, 305-308.

21. Blessing, E.; Campbell, L.A.; Rosenfeld, M.E.; Chough, N.; Kuo, C.C. Chlamydia pneumoniae infection accelerates hyperlipidemia induced atherosclerotic lesion development in $\mathrm{C} 57 \mathrm{BL} / 6 \mathrm{~J}$ mice. Atherosclerosis 2001, 158, 13-17.

22. Sessa, R.; Nicoletti, M.; di Pietro, M.; Schiavoni, G.; Santino, I.; Zagaglia, C.; del Piano, M.; Cipriani, P. Chlamydia pneumoniae and atherosclerosis: Current state and future prospectives. Int. J. Immunopathol. Pharmacol. 2009, 22, 9-14.

23. Chen, S.; Shimada, K.; Zhang, W.; Huang, G.; Crother, T.R.; Arditi, M. IL-17A is proatherogenic in high-fat diet-induced and Chlamydia pneumoniae infection-accelerated atherosclerosis in mice. J. Immunol. 2010, 185, 5619-5627.

24. Huang, C.Y.; Shih, C.M.; Tsao, N.W.; Chen, Y.H.; Li, C.Y.; Chang, Y.J.; Chang, N.C.; Ou, K.L.; Lin, C.Y.; Lin, Y.W.; et al. GroEL1, from Chlamydia pneumoniae, induces vascular adhesion molecule 1 expression by p37(AUF1) in endothelial cells and hypercholesterolemic rabbit. PLoS One 2012, 7, e42808.

25. Gaydos, C.A. Growth in vascular cells and cytokine production by Chlamydia pneumoniae. J. Infect. Dis. 2000, 181, S473-S478.

26. Netea, M.G.; Kullberg, B.J.; Galama, J.M.; Stalenhoef, A.F.; Dinarello, C.A.; van der Meer, J.W. Non-LPS components of Chlamydia pneumoniae stimulate cytokine production through Toll-like receptor 2-dependent pathways. Eur. J. Immunol. 2002, 32, 1188-1195.

27. Yang, X.; Coriolan, D.; Schultz, K.; Golenbock, D.T.; Beasley, D. Toll-like receptor 2 mediates persistent chemokine release by Chlamydia pneumoniae-infected vascular smooth muscle cells. Arterioscler. Thromb. Vasc. Biol. 2005, 25, 2308-2314.

28. Högdahl, M.; Söderlund, G.; Kihlström, E. Expression of chemokines and adhesion molecules in human coronary artery endothelial cells infected with Chlamydia (Chlamydophila) pneumoniae. APMIS 2008, 116, 1082-1088.

29. Al-Bannawi, A.; Al-Wesebai, K.; Taha, S.; Bakhiet, M. Chlamydia pneumoniae induces chemokine expression by platelets in patients with atherosclerosis. Med. Princ. Pract. 2011, 20, $438-443$. 
30. Wang, A.; Al-Kuhlani, M.; Johnston, S.C.; Ojcius, D.M.; Chou, J.; Dean, D. Transcription factor complex AP-1 mediates inflammation initiated by Chlamydia pneumoniae infection. Cell Microbiol. 2013, 15, 779-794.

31. Roivainen, M.; Viik-Kajander, M.; Palosuo, T.; Toivanen, P.; Leinonen, M.; Saikku, P.; Tenkanen, L.; Manninen, V.; Hovi, T.; Mänttäri, M. Infections, inflammation, and the risk of coronary heart disease. Circulation 2000, 101, 252-257.

32. Schiavoni, G.; di Pietro, M.; Ronco, C.; de Cal, M.; Cazzavillan, S.; Rassu, M.; Nicoletti, M.; del Piano, M.; Sessa, R. Chlamydia pneumoniae infection as a risk factor for accelerated atherosclerosis in hemodialysis patients. J. Biol. Regul. Homeost. Agents 2010, 24, 367-375.

33. Swierszcz, J.; Jacek, D.S.; Milewicz, T.; Krzysiek, J.; Sztefko, K.; Galicka-Latała, D. One-year observation of inflammatory markers in patients with aortic valve stenosis who expressed high or low Chlamydia pneumoniae antibody titers. J. Heart Valve Dis. 2012, 21, 599-607.

34. Di Pietro, M.; Filardo, S.; de Santis, F.; Sessa, R. Chlamydia pneumoniae infection in atherosclerotic lesion development through oxidative stress: A brief overview. Int. J. Mol. Sci. 2013, 14, 15105-15120.

35. Yaraei, K.; Campbell, L.A.; Zhu, X.; Liles, W.C.; Kuo, C.C.; Rosenfeld, M.E. Chlamydia pneumoniae augments the oxidized low-density lipoprotein-induced death of mouse macrophages by a caspase-independent pathway. Infect. Immun. 2005, 73, 4315-4322.

36. Nazzal, D.; Cantero, A.V.; Therville, N.; Segui, B.; Negre-Salvayre, A.; Thomsen, M.; Benoist, H. Chlamydia pneumoniae alters mildly oxidized low-density lipoprotein-induced cell death in human endothelial cells, leading to necrosis rather than apoptosis. J. Infect. Dis. 2006, 193, 136-145.

37. Stocker, R.; Keaney, J.F., Jr. New insights on oxidative stress in the arterial wall. J. Thromb. Haemost. 2005, 3, 1825-1834.

38. Li, H.; Horke, S.; Förstermann, U. Oxidative stress in vascular disease and its pharmacological prevention. Trends Pharmacol. Sci. 2013, 34, 313-319.

39. Kälvegren, H.; Bylin, H.; Leanderson, P.; Richter, A.; Grenegård, M.; Bengtsson, T. Chlamydia pneumoniae induces nitric oxide synthase and lipoxygenase-dependent production of reactive oxygen species in platelets. Effects on oxidation of low density lipoproteins. Thromb. Haemost. 2005, 94, 327-335.

40. Azenabor, A.A.; Yang, S.; Job, G.; Adedokun, O.O. Elicitation of reactive oxygen species in Chlamydia pneumoniae-stimulated macrophages: $\mathrm{A} \mathrm{Ca}^{2+}$-dependent process involving simultaneous activation of NADPH oxidase and cytochrome oxidase genes. Med. Microbiol. Immunol. 2005, 194, 91-103.

41. Azenabor, A.A.; Muili, K.; Akoachere, J.F.; Chaudhry, A. Macrophage antioxidant enzymes regulate Chlamydia pneumoniae chronicity: Evidence of the effect of redox balance on host-pathogen relationship. Immunobiology 2006, 211, 325-339.

42. Kreutmayer, S.; Csordas, A.; Kern, J.; Maass, V.; Almanzar, G.; Offterdinger, M.; Öllinger, R.; Maass, M.; Wick, G. Chlamydia pneumoniae infection acts as an endothelial stressor with the potential to initiate the earliest heat shock protein 60-dependent inflammatory stage of atherosclerosis. Cell Stress Chaperones 2013, 18, 259-268. 
43. Chen, C.; Chai, H.; Wang, X.; Lin, P.H.; Yao, Q. Chlamydia heat shock protein 60 decreases expression of endothelial nitric oxide synthase in human and porcine coronary artery endothelial cells. Cardiovasc. Res. 2009, 83, 768-777.

44. Mueller, K.E.; Wolf, K. C. pneumoniae disrupts eNOS trafficking and impairs NO production in human aortic endothelial cells. Cell Microbiol. 2014, doi:10.1111/cmi.12341.

45. Lin, Y.W.; Huang, C.Y.; Chen, Y.H.; Shih, C.M.; Tsao, N.W.; Lin, C.Y.; Chang, N.C.; Tsai, C.S.; Tsai, H.Y.; Tsai, J.C.; et al. GroEL1, a heat shock protein 60 of Chlamydia pneumoniae, impairs neovascularization by decreasing endothelial progenitor cell function. PLoS One 2013, 8, e84731.

46. Lin, F.Y.; Lin, Y.W.; Huang, C.Y.; Chang, Y.J.; Tsao, N.W.; Chang, N.C.; Ou, K.L.; Chen, T.L.; Shih, C.M.; Chen, Y.H. GroEL1, a heat shock protein 60 of Chlamydia pneumoniae, induces lectin-like oxidized low-density lipoprotein receptor 1 expression in endothelial cells and enhances atherogenesis in hypercholesterolemic rabbits. J. Immunol. 2011, 186, 4405-4414.

47. Rivera, J.; Walduck, A.K.; Strugnell, R.A.; Sobey, C.G.; Drummond, G.R. Chlamydia pneumoniae induces a pro-inflammatory phenotype in murine vascular smooth muscle cells independently of elevating reactive oxygen species. Clin. Exp. Pharmacol. Physiol. 2012, 39, 218-226.

48. Deniset, J.F.; Pierce, G.N. Possibilities for therapeutic interventions in disrupting Chlamydophila pneumoniae involvement in atherosclerosis. Fundam. Clin. Pharmacol. 2010, 24, 607-617.

49. Campbell, L.A.; Rosenfeld, M.E.; Persistent, C. pneumoniae infection in atherosclerotic lesions: Rethinking the clinical trials. Front. Cell. Infect. Microbiol. 2014, 4, 34.

50. Deby-Dupont, G.; Mouithys-Mickalad, A.; Serteyn, D.; Lamy, M.; Deby, C. Resveratrol and curcumin reduce the respiratory burst of Chlamydia-primed THP-1 cells. Biochem. Biophys. Res. Commun. 2005, 333, 21-27.

51. Di Pietro, M.; de Santis, F.; Schiavoni, G.; Filardo, S.; Sessa, R. Resveratrol in Chlamydia pneumoniae induced foam cell formation and interleukin-17A sythesis. J. Biol. Regul. Homeost. Agents 2013, 27, 509-518.

52. Mouithys-Mickalad, A.; Deby-Dupont, G.; Dogne, J.M.; de Leval, X.; Kohnen, S.; Navet, R.; Sluse, F.; Hoebeke, M.; Pirotte, B.; Lamy, M. Effects of COX-2 inhibitors on ROS produced by Chlamydia pneumoniae-primed human promonocytic cells (THP-1). Biochem. Biophys. Res. Commun. 2004, 325, 1122-1130.

53. Antonopoulos, A.S.; Margaritis, M.; Shirodaria, C.; Antoniades, C. Translating the effects of statins: from redox regulation to suppression of vascular wall inflammation. Thromb. Haemost. 2012, 108, 840-848.

54. Kothe, H.; Dalhoff, K.; Rupp, J.; Müller, A.; Kreuzer, J.; Maass, M.; Katus, H.A. Hydroxymethylglutaryl coenzyme A reductase inhibitors modify the inflammatory response of human macrophages and endothelial cells infected with Chlamydia pneumoniae. Circulation 2000, 101, 1760-1763.

55. Dechend, R.; Gieffers, J.; Dietz, R.; Joerres, A.; Rupp, J.; Luft, F.C.; Maass, M. Hydroxymethylglutaryl coenzyme A reductase inhibition reduces Chlamydia pneumoniae-induced cell interaction and activation. Circulation 2003, 108, 261-265. 
56. Prochnau, D.; Rödel, J.; Prager, K.; Kuersten, D.; Heller, R.; Straube, E.; Figulla, H.R. Induced expression of lectin-like oxidized LDL receptor-1 in vascular smooth muscle cells following Chlamydia pneumoniae infection and its down-regulation by fluvastatin. Acta Microbiol. Immunol. Hung. 2010, 57, 147-155.

57. Kang, D.H.; Kang, S.W. Targeting cellular antioxidant enzymes for treating atherosclerotic vascular disease. Biomol. Ther. 2013, 21, 89-96.

58. Fukuoka, K.; Sawabe, A.; Sugimoto, T.; Koga, M.; Okuda, H.; Kitayama, T.; Shirai, M.; Komai, K.; Komemushi, S.; Matsuda, K. Inhibitory actions of several natural products on proliferation of rat vascular smooth muscle cells induced by Hsp60 from Chlamydia pneumoniae J138. J. Agric. Food Chem. 2004, 52, 6326-6329.

59. Bjelakovic, G.; Nikolova, D.; Gluud, C. Antioxidant supplements and mortality. Curr. Opin. Clin. Nutr. Metab. Care 2014, 17, 40-44.

60. Berger, R.G.; Lunkenbein, S.; Ströhle, A.; Hahn, A. Antioxidants in food: mere myth or magic medicine? Crit. Rev. Food Sci. Nutr. 2012, 52, 162-171.

61. Brackman, G.; Cos, P.; Maes, L.; Nelis, H.J.; Coenye, T. Quorum sensing inhibitors increase the susceptibility of bacterial biofilms to antibiotics in vitro and in vivo. Antimicrob. Agents Chemother. 2011, 55, 2655-2661.

62. Duncan, M.C.; Linington, R.G.; Auerbuch, V. Chemical inhibitors of the type three secretion system: disarming bacterial pathogens. Antimicrob. Agents Chemother. 2012, 56, 5433-5441.

63. Beeckman, D.S.; de Puysseleyr, L.; de Puysseleyr, K.; Vanrompay, D. Chlamydial biology and its associated virulence blockers. Crit. Rev. Microbiol. 2014, 40, 313-328.

64. Byrne, G.I.; Kalayoglu, M.V. Chlamydia pneumoniae and atherosclerosis: links to the disease process. Am. Heart J. 1999, 138, S488-S490.

65. Sasu, S.; LaVerda, D.; Qureshi, N.; Golenbock, D.T.; Beasley, D. Chlamydia pneumoniae and chlamydial heat shock protein 60 stimulate proliferation of human vascular smooth muscle cells via toll-like receptor 4 and p44/p42 mitogen-activated protein kinase activation. Circ. Res. 2001, $89,244-250$.

66. Da Costa, C.U.; Wantia, N.; Kirschning, C.J.; Busch, D.H.; Rodriguez, N.; Wagner, H.; Miethke, T. Heat shock protein 60 from Chlamydia pneumoniae elicits an unusual set of inflammatory responses via Toll-like receptor 2 and 4 in vivo. Eur. J. Immunol. 2004, 34, 2874-2884.

67. Jha, H.C.; Srivastava, P.; Prasad, J.; Mittal, A. Chlamydia pneumoniae heat shock protein 60 enhances expression of ERK, TLR-4 and IL-8 in atheromatous plaques of coronary artery disease patients. Immunol. Investig. 2011, 40, 206-222.

(C) 2014 by the authors; licensee MDPI, Basel, Switzerland. This article is an open access article distributed under the terms and conditions of the Creative Commons Attribution license (http://creativecommons.org/licenses/by/4.0/). 